\title{
Redistribution Measurement: A New (Old) Tool for Finding Heart Disease?
}

\author{
Richard M Fleming1*, Matthew R Fleming ${ }^{1}$, Tapan K Chaudhuri ${ }^{2}$ and Gordon M Harrington ${ }^{3}$ \\ ${ }^{1}$ FHHI-OmnificImaging-Camelot, Los Angeles, CA, USA \\ ${ }^{2}$ Eastern Virginia Medical School, Norfolk, Virginia, USA \\ ${ }^{3}$ University of Northern Iowa, Cedar Falls, Iowa, USA \\ *Corresponding Author: Richard M Fleming, FHHI-OmnificImaging-Camelot, Los Angeles, CA, USA.
}

Received: September 04, 2019; Published: September 12, 2019

DOI: 10.31080 /ASPS.2019.03.0394

\begin{abstract}
Background: Myocardial perfusion imaging (MPI) has been used for decades to look for evidence of ischemia and infarction. Several limitations to MPI have been identified including qualitative interpretation leading to sensitivity and specificity errors, loss of data due to lack of quantitative camera calibration, and failure to understand the redistribution properties of several isotopes. This study corrects for these errors by quantitatively calibrating nuclear cameras, measuring isotope redistribution properties and comparing these results to ischemia, infarction and the combined effect of flow and cellular viability - viz. myocardial "condition".

Methods: Utilizing a new utility patent, The Fleming Method for Tissue and Vascular Differentiation and Metabolism (FMTVDM; patent \# 9566037), forty-one veterans admitted for evaluation of heart disease, were studied. The results were compared with quantified coronary arteriography (QCA), electrocardiograms and cardiac enzymes.

Results: Parabolic regression of a rating of infarction on FMTVDM showed high predictability, $\mathrm{R}(\mathrm{CI} 95 \%)=0.71$ to $0.91,(\mathrm{P}=4.7 \times$ $10^{-12}$ ). Within the 22 patient subset, parabolic regression of infarction on redistribution (washout) yielded an effect size of R (CI95\%) $=0.77$ to $0.96,\left(\mathrm{P}=4.0 \times 10^{-9}\right)$. Regression of stenosis on washout yielded an effect size of $\mathrm{R}(\mathrm{CI} 95 \%)=0.72$ to $0.95,\left(\mathrm{P}=3.8 \times 10^{-8}\right)$. $\mathrm{A}$ combined measure of Myocardial Condition showed comparable effects, $\mathrm{R}(\mathrm{CI} 95 \%)=0.81$ to $0.96,\left(\mathrm{P}=5.4 \times 10^{-10}\right)$.

Conclusions: Quantification of isotope redistribution, measures two fundamental properties not possible using qualitative methods or anatomic measures of heart disease. The first is regional blood flow difference (RBFD), which produces ischemia and consequently angina. The second is cellular viability and the consequential ability of myocytes to take up and retain isotopes. As coronary artery disease develops, it first impairs coronary flow reserve (CFR) and later results in impaired myocyte function. Quantitative measurement looking at both flow and function simultaneously provides a more in-depth understanding of the level of cardiac health - viz. cardiac condition.
\end{abstract}

Keywords: FMTVDM; Isotope Redistribution Measurement; Ischemia; Infarction; Condition

\section{Introduction}

Nuclear myocardial perfusion imaging (MPI) diagnosis of ischemia centers on a single stress image of a heart vasodilated by exercise or chemical stressors. A rest image may also be obtained. Historically much controversy followed sequential stress imaging, which found instances of redistribution (washout, fill-in) with the radioactive tracer, thallium-201, after 4 hours but not after 2 hours $[1,2]$. The presence or absence of fill-in was attributed to cell viability and thus differentiated stenosis and infarction. Though sequential imaging for redistribution remains in the CPT codes, these results ceased to be considered relevant because they rested on canine models and because contemporary tracers, such as technetium-99m sestamibi, are reportedly not subject to redistribution. 
Current standard physician protocols from joint medical societies [3] and patient explanatory instruction sheets [4] make no reference to sequential images. The technical data, provided to users by the nuclear imaging equipment and radiopharmaceutical manufacturers [5,6], explain: (1) that $92 \%$ of the tracer is taken up and thus removed from the blood stream within 5 minutes after injection, (2) there it is held in an essentially static state in proportion to cell health, and (3) it is not redistributed among cells nor released back into the blood stream.

Clearance of the tracer is reported as accomplished through the hepatobiliary system, reaching $60 \%$ in 48 hours. No explanation has been proffered to explain how this clearance can occur without tracer return to the bloodstream. Inconsistent with the generally accepted view, a few studies have reported quantitative variations in successive stress images, both washout and wash-in [7-14]. No explanation has been offered though occasional comments suggest these variations might have some unknown clinical relevance.

As an alternative to the essentially static "uptake/retention" theory we offer and test an alternative dynamic, continuing "uptake/release", hypothesis. From this perspective, uptake is a function of isotope availability (regional myocardial blood flow) and cellular health. Retention is transient with release of the isotope back into the blood stream or redistribution to other cells, a function of cellular health and re-uptake subject to a cellular refractory period.

With the static uptake/retention model, heart condition can be ascertained by visually examining the gray-scale or color-scale nuclear image, showing relative levels of radioactivity. The dynamic uptake/release model utilizes the quantitative features of the nuclear camera to make quantitative comparisons between successive images. In essence, the procedural logic is to create a physiologic specimen by injection and then make successive imaging examinations with no intervening events, which might affect the specimen, other than the passage of time. We consider the diagnostic implications of any observable changes other than radioactive decay.

\section{Materials and Methods}

Current hospital case records were examined for 41 cardiology patients who had myocardial perfusion, single photon emission computed tomography, using conventional "stress-rest" protocols
[15] with the addition of another 5-minute image acquired following "stress", which was quantitatively compared with the other stress image. Images were reconstructed by a single technician on either of two nuclear cameras (Philips or Picker), under the direction of any of several physicians using either sestamibi or myoview as the injected isotope. A stress effect was created by injection of adenosine or dobutamine [15]. Severity of infarction was rated as 0,1 , or 2 for anterior, inferior and lateral regions from rest images. No isotope uptake was rated as 2 , and limited uptake as 1 . The sum of the two provided a 0-6 rating for Infarction.

The mean quantitative percent diameter stenosis from these regions obtained by catheterization was the measure of stenosis. Stenosis data were available for a subset of 22 patients whom we compare in detail. For a global index of Myocardial Condition the sigma weighted Infarction and Stenosis measures were summed and reported in units of standard deviation. The percent reduction in radioactivity for the total heart between 5 minutes and one hour after injection minus $10 \%$ for 55 minutes of radioactive decay was designated as Washout.

Diagnostic inference from Washout was evaluated by least squares regression of Infarction on Washout, of Stenosis on Washout, and of Myocardial Condition on Washout fitting the hypothesized model, $\mathrm{y}=\mathrm{cx}^{2}$.

\section{Results}

When the results of all 41 patients are analyzed, looking at infarction data, the most conservative fit is $y=\mathrm{cx}^{2}$. Confidence intervals replace null hypothesis significance testing. For the full sample $(\mathrm{n}=41):$ Infarction $=0.00082 \times$ Washout $^{2}, \mathrm{~F}=94.0, \mathrm{df}(1,40),(\mathrm{P}=$ $\left.4.7 \times 10^{-12}\right), \mathrm{R}(\mathrm{CI} 95 \%)=0.71$ to 0.91 .

Figure one shows the subset $(\mathrm{n}=22)$ for which independent catheterization stenosis data were available in addition to infarction data: (a) Infarction $=0.0011 \times$ Washout $^{2}, \mathrm{~F}=92.0$, df $(1,21)$, $\left(\mathrm{P}=4.0 \times 10^{-9}\right), \mathrm{R}(\mathrm{CI} 95 \%)=0.77$ to 0.96 , (b) Stenosis $=0.011 \times$ Washout $^{2}, \mathrm{~F}=70.4$, df $(1,21),\left(\mathrm{P}=3.8 \times 10^{-8}\right), \mathrm{R}(\mathrm{CI} 95 \%)=0.72$ to 0.95, and (c) a composite measure, Myocardial Condition $=0.0011$ $\times$ Washout $^{2}, \mathrm{~F}=115.5, \mathrm{df}(1,21),\left(\mathrm{P}=5.4 \times 10^{-10}\right), \mathrm{R}(\mathrm{CI} 95 \%)=0.81$ to 0.96 . Graphic bands show the standard error of the fit.

Infarction and stenosis were not statistically correlated in this sample: $r($ CI95\%) $=-0.05$ to 0.69 .
Infarction $=0.0010 \cdot$ Washout $^{2}$

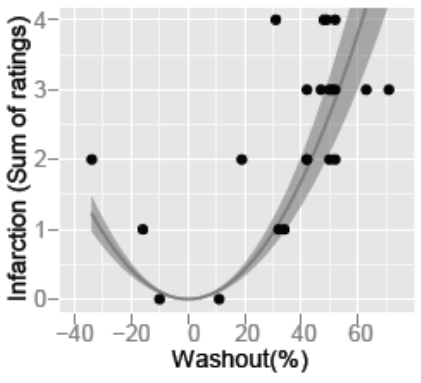

Stenosis $=0.011 \cdot$ Washout $^{2}$

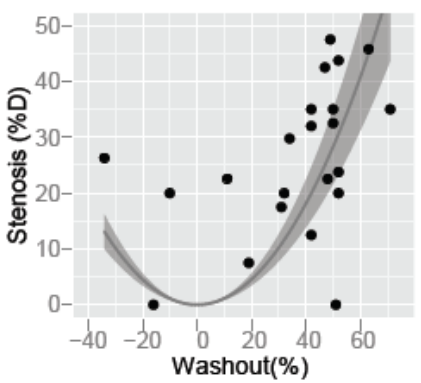

\section{Condition $=0.0010 \cdot$ Washout $^{2}$}

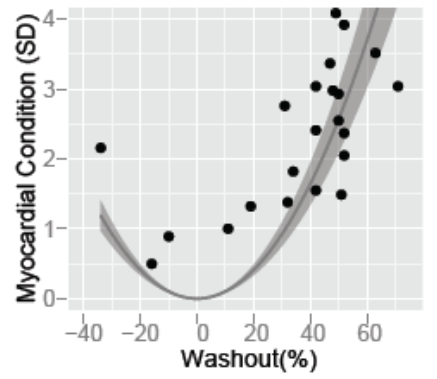




\section{Discussion}

Both isotope uptake and release are dependent on cellular functions, which are probably not independent within the cell. Uptake is also dependent on blood flow so the two factors are not statistically independent and their resultant must be curvilinear and of at least second degree (parabolic). Second-degree functions with redistribution (wash-in and washout) as the predictor provide inference to Infarction and Stenosis. Adjusting for radioactive decay provides a biological parametric zero, eliminating any intercept or first-degree term.

Though redistribution assesses stenosis and infarction with a common parabolic function, infarction is a cell viability function while stenosis is a blood flow function. Thus, there is little reason to expect infarction and stenosis to be significantly correlated and they are not. A definitive test of their independence would require a much larger sample. The model does not enable us to discriminate which function is influencing the outcome but rather provides an index of myocardial condition. The fit of the composite measure is an index of how well it performs that function.

Approximately ten percent of the patients showed a slowed uptake (redistribution) of the isotope. Three patients showed substantial redistribution wash-in; an initial impaired uptake by myocytes at 5-minutes post-stress, which increased over time, appearing "visually" normal during the 60-minute post-stress imaging. These visually normal 60-minute post stress nuclear images were screened as normal studies. Note that the data of this study are from patient records and the judgments of normality were those of the patients' cardiologists, not of the authors. Subsequently these patients were catheterized only because they unexpectedly had acute cardiac episodes. The degree of wash-in in a fourth patient differed only trivially from zero and was not accompanied by infarction or stenosis.

Visual interpretation of nuclear heart images derives from the contrast between healthy and diseased regions of the myocardium in their isotope retention. The uptake/release model suggests some individuals could have very slow uptake in the presence of substantial occlusion of myocardial blood flow. This could reduce the differential between uptake and release, reducing or eliminating contrast thus masking visual evidence of disease. Contrast could be reduced to the point of apparent normalcy. A uniform quantitative reduction would not be detected visually, since the visual image screen brightness is normally adjusted by the tech- nician for maximum readability. Additionally, a substantial reduction in myocardial blood flow can be interpreted as symptomatic of cardiovascular disease. With sufficiently slow uptake, uptake could peak after release has peaked, presenting a visually normal contrast image and a negative washout (wash-in). Wash-in provides a valuable diagnostic cue of critical underlying disease.

\section{Conclusion}

Quantitative differences [14] between two images taken at different points in time following a single stress injection (redistribution) are strongly diagnostic of coronary artery disease, myocyte function and the combined myocardial condition. Redistribution data provided a non-invasive quantitative measure, including identification of critical health status which otherwise would have gone undetected.

\section{Disclosure}

COI: FMTVDM patent was issued to first author. No funding to report. Any material reproduction provided through expressed consent of first author.

\section{Bibliography}

1. Pohost GM., et al. "Differentiation of transiently ischemic from infarcted myocardium by serial imaging after a single dose of thallium-201". Circulation 55.2 (1977): 294-302.

2. van der Wall EE and Schwaiger M. "Twenty years of myocardial perfusion imaging 1976-1996: anything new for the clinical cardiologist?". European Heart Journal 17.7 (1996): 987-990.

3. Anagnostopoulos C., et al. "Procedure guidelines for radionuclide myocardial perfusion imaging”. Heart 90 (2004): 1-10.

4. National Institutes of Health. "Persantine sestamibi scan: Outpatient instruction sheet” (2006).

5. Bristol-Myers Squibb Medical Imaging. "Kit for the Preparation of Technetium Tc99m Sestamibi for Injection". Billerica, MA: Bristol-Myers Squibb Medical Imaging (2003).

6. General Electric Company. "Nuclear myocardial perfusion imaging. Medcyclopaedia: General Electric Company" (2008).

7. Ayalew A., et al. "A comparison of the overall first-pass kinetics of thallium-201 and technetium-99m MIBI in normoxic and low-flow ischaemic myocardium". European Journal of Nuclear Medicine 27.11 (2000): 1632-1640. 
8. Fujiwara S., et al. "Prediction of functional recovery in acute myocardial infarction: comparison between sestamibi reverse redistribution and sestamibi/BMIPP mismatch". Journal of Nuclear Cardiology 5.2 (1998): 119-127.

9. Liu Z., et al. "Detection of myocardial viability in ischemic-reperfused rat hearts by Tc-99m sestamibi kinetics". Journal of Nuclear Cardiology 8.6 (2001): 677-686.

10. Richter WS., et al. "Washout and redistribution between immediate and two-hour myocardial images using technetium99m sestamibi". European Journal of Nuclear Medicine 22.1 (1995): 49-55.

11. Takahashi N., et al. "Myocardial uptake of $99 \mathrm{mTc}$ - tetrofosmin, sestamibi, and $201 \mathrm{Tl}$ in a model of acute coronary reperfusion". Circulation 94.10 (1996): 2605-2613.

12. Tanaka R., et al. "Clinical implication of reverse redistribution on $99 \mathrm{mTc}$-sestamibi images for evaluating ischemic heart disease". Annals of Nuclear Medicine 20.5 (2006): 349-356.

13. Fleming RM., et al. "USVAH Study demonstrates statistically significant improvement in diagnosis and care of U.S. Veterans using FMTVDM-FHRWW ${ }^{\oplus \circledast ~ " Q u a n t i t a t i v e " ~ N u c l e a r ~ I m a g i n g . ~}$ The era of truly quantitative stress-first, stress-only imaging has begun!". Journal of Nuclear Medicine and Radiation Therapy (2018).

14. Fleming RM., et al. "Multi Center Clinical Trial Confirms FMTVDM $^{\odot \oplus}$ MPI in Seven Modern Clinical Laboratories in the U.S.A. and Asia. Artificial Intelligence (AI) with True Quantification". Journal of Nuclear Medicine and Radiation Therapy 9.4 (2018).

15. Fleming RM. “Chapter 31. Nuclear Cardiology: Its Role in the Detection and Management of Coronary Artery Disease Textbook of Angiology". John C. Chang Editor, Springer-Verlag New York (1999): 397-406.

\section{Volume 3 Issue 10 October 2019}

(C) All rights are reserved by Richard M Fleming., et al. 